\title{
Asymmetric exclusion processes on a closed network with bottlenecks
}

\author{
Rakesh Chatterjee, Anjan Kumar Chandra and Abhik Basu \\ E-mail: rakesh.chatterjee@saha.ac.in, anjanphys@gmail.com, \\ abhik. basu@saha.ac.in \\ Condensed Matter Physics Division, Saha Institute of Nuclear Physics, Kolkata \\ 700064, India
}

\begin{abstract}
We study the generic nonequilibrium steady states in asymmetric exclusion processes on a closed network with bottlenecks. To this end we proposes and study closed simple networks with multiply-connected non-identical junctions. Depending upon the parameters that define the network junctions and the particle number density, the models display phase transitions with both static and moving density inhomogeneities. The currents in the models can be tuned by the junction parameters. Our models highlight how extended and point defects may affect the density profiles in a closed directed network. Phenomenological implications of our results are discussed.
\end{abstract}

PACS numbers: 05.60.Cd, 89.75.-k, 02.50.-r 


\section{Introduction}

Simplest physical modeling of directed or active classical transports in one dimensions (1D) (e.g., along narrow channels) are often provided in terms of asymmetric simple exclusion processes. A well-known example of directed transport in 1D is the system of unidirectionally moving vehicular traffic along roads [1,2]. Narrow roads with excluded volume interactions between vehicles having no possibility of overtaking, are welldescribed by the totally asymmetric simple exclusion process (TASEP) [3]. Important phenomenological questions about traffic networks include how defects (both extended and point) along a road may control the density profiles and currents in the steady states. We concern ourselves in exploring these issues in terms of a simple model in this paper.

In order to focus on the essential physics of the system, we consider a minimal closed directed network (hereafter Model I) consisting of just three segments, with each executing TASEP, two of them (marked $T_{A}$ and $T_{B}$ in Fig. 1) being parallelly and the third one $\left(T_{C}\right)$ anti-parallelly attached to the multiply connected left $(L J)$ and right $(R J)$ junctions. Junctions $L J$ and $R J$ are non-identical, since the system is directed. Notice that $T_{C}$, the antiparallel TASEP channel may be viewed as an extended defect or an extended bottleneck in the system, since its maximum current carrying capacity is less than the total maximum current carrying capacities of the two parallel channels. Thus, $T_{C}$ restricts the maximum permissible system current. Therefore, $T_{C}$ acts like an (extended) bottleneck in the system. While in general the length of an extended defect is not necessarily same as the lengths of either of the parallel channels, but for simplicity we take all of them to have the same length with equal number of lattice sites. Although the branching of particles at $L J$ is controlled by a parameter $\theta_{L}$, at $R J$ there is no analogous control parameter.

There have been a number of applications of TASEP and TASEP-like models in studies on directed vehicular traffic along roads, studying various physical aspects of traffic jam described as TASEP or TASEP-like systems. For instance, the NagelSchreckenberg model, closely related to TASEP, has been proposed as a theoretical description for freeway traffic. The model displays traffic jam when the vehicle density is high [4]. Shock propagation in traffic systems has also been studied using TASEP [5,6]. In a recent work, Ref. [7] has studied interactions between vehicles and pedestrians in terms of a TASEP with a bottleneck at a boundary caused by interactions. Furthermore, Ref. [8] has considered an "optimal velocity model" with two kinds of vehicles (fast and slow). With specific lane change rules and under periodic boundary conditions, the traffic states change with increasing densities. Further, Ref. [8] finds a new phenomenon of ratio inversion. In a 1D traffic model, Ref. [9] studies the impact of disruptions on road networks, and the recovery process after the disruption is removed from the system. Extensive reviews on applications of TASEP-like models in the studies of vehicular traffic and related areas are available in Refs. [1,2]. Our studies on Model I are complementary to these model studies. We have strict particle number conservation and an extended 
defect. Thus Model I should be useful for studying traffic problems where the vehicle current is controlled by an extended defect for a fixed number of vehicles. In the Appendix A of this article we discuss an extension of Model I, by including additional junction parameters $\theta_{R A}$ and $\theta_{R B}$ that control the relative flow of particles from $T_{A}$ and $T_{B}$ to $T_{C}$ at the junction $R J$, which appear as point defects in the model, can be considered as traffic signals at that junction. We call this Model II; see Fig. A1. This further allows us to find how the steady state densities are affected by the point defects at $R J$. Our results reveal interesting interplay between the extended and point defects, which ultimately controls the steady state densities.

We now compare Model I with a recently proposed model by us [10], where two asymmetric exclusion processes in the form of two TASEPs are coupled with $1 D$ diffusive motion or SEP. The principal physical difference between them is that, the diffusive channel in Ref. [10] does not control the maximum current in the model, allowing each of the TASEP channels there to reach their individual maximum currents. In contrast, the maximum current in the present models is limited by $T_{C}$, the extended bottleneck, which is absent in Ref. [10]. As we shall see below, the extended bottleneck has major consequences on the phases and density profiles in Model I; some phases individually accessible to an open TASEP or accessed by the model of Ref. [10, are ruled out here.

Here is a summary of our specific results. Both Model I and Model II display generic nonequilibrium phase transitions associated with a variety of density profiles ranging from uniform (flat) profiles to localised (LDW) or static and delocalised (DDW) or moving domain walls, controlled by the particle number and the junction parameters. Nonetheless, there are significant differences between the density profiles of Model I and Model II. Due to the absence of any point defect at $R J$, the DDWs in $T_{A}$ and $T_{B}$ in Model I are always overlapping, a consequence of a special symmetry at the delocalisation point; in contrast, the DDWs do not overlap in Model II. Furthermore, the presence of the point defect at $R J$ in Model II allows for a class of phases, which are not permitted in Model I. For example, in Model II, the extended bottleneck $T_{C}$ can be in its LD-HD coexistence or LDW phase, whereas such a possibility is ruled out in Model I on the ground of current conservation and symmetry. On the whole, our models here serve as good candidates to study the interplay between extended and point defects, and number conservation in closed, simple, directed networks. Our calculational framework may be systematically extended to a network with a larger number of segments/joints. Despite the simplicity and the minimalist nature of our models, the above results should be potentially relevant in the contexts of defect-controlled vehicular traffic in closed network of roads. The rest of the paper is organised as follows. In Sec. 2 we construct Model I. In Sec. 3 we obtain the steady state density profiles and the phase diagram of Model I. In Sec. 4 we conclude. In Appendix A we introduce Model II. The corresponding phase diagram and the density profiles are analysed, respectively, in Appendix B and Appendix C. 


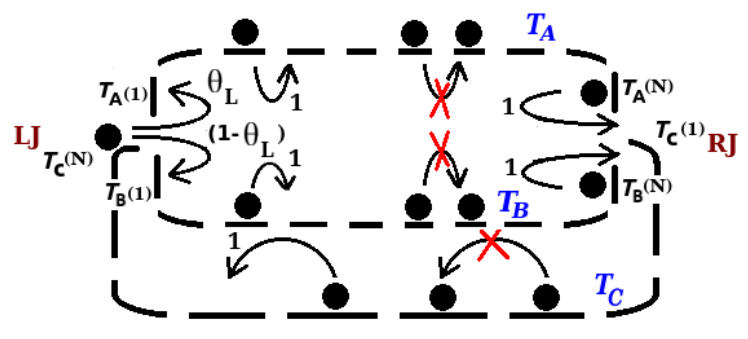

Figure 1. (Color online) Schematic diagram of the Model I; $L J / R J$ refer to the left/right junctions. Site labels run from $i=1$ to $N$ from $L J$ to $R J$ for $T_{A, B}$ and for $T_{C}$ from $R J$ to $L J$.

\section{Construction of Model I}

In Model I, each channel consists of equal number of sites designated by $i=1,2, \ldots N$. As shown in Fig. 1, the left (right) most site of $T_{A}$ is labeled as $T_{A}(1)\left(T_{A}(N)\right)$. Similar labeling follow for $T_{B}$ and $T_{C}$. Particles from $T_{C}$ enter at the left end of $T_{A}\left(T_{B}\right)$ if empty with probability $\theta_{L}\left(1-\theta_{L}\right)$ and exit to the right end of $T_{C}$ if vacant from the right end of $T_{A}$ and $T_{B}$ with rate unity. In each of $T_{A}, T_{B}\left(T_{C}\right)$ particles can only hop with rate unity to the right (left) neighbor if it is empty. The global particle density is $n_{p}=N_{p} / 3 N$, with $N_{p}$ being the total particle number in the system. In this model, the phases are parametrized and tunable by $n_{p}$ and $\theta_{L}$. The presence of $L J$ and $R J$ breaks the translational invariance, and hence, non-trivial steady states are expected [11].

\section{Steady state density profiles}

We use mean-field theory (MFT) together with extensive Monte-Carlo simulation (MCS) by using random sequential updates to obtain the steady state density profiles in our model. In the MFT, the system is considered as a collection of three separate TASEP channels with effective entry and exit rates [11,12] $\left(\alpha_{m}\right.$ and $\beta_{m}$, respectively for channel $\left.T_{m}, m=A, B, C\right)$, to be determined by applying the condition of constancy of particle currents at the junctions $L J$ and $R J$ and in the bulk. These immediately allow us to obtain the phases of the individual channels and hence of the whole model in terms of the known results for TASEP with open boundaries. In the discrete lattice description of our model, we denote the density at a particular site $i$ in channel $T_{m}$ by $\rho_{m}^{i}=\left\langle n_{m}^{i}\right\rangle$, $m=A, B, C, n_{m}^{i}=0$ or 1 and $\langle\ldots\rangle$ denotes time and configuration averages, whereas in MFT considering continuum limit the density is defined as $\rho_{m}(x)$, where $x=i / N$, and in the thermodynamic limit (TL) $N \gg 1, x$ in the range $0 \leq x \leq 1$. Given the symmetry between $T_{A}$ and $T_{B}, \rho_{A}(x)$ and $\rho_{B}(x)$ interchange when $\theta_{L}$ interchanges with $\left(1-\theta_{L}\right)$. This symmetry is identical to the one in the model of Ref. [10].

Recall that an isolated TASEP can be in four different phases in its steady states: Low density (LD), high density (HD), maximal current (MC) and coexistence or domain wall (DW) phases [13,14]; clearly there are almost 64 possibilities for the overall density profiles of our model. For the ease of notation and compactness, we denote a phase by 
(X-Y-Z) where $\mathrm{X} / \mathrm{Y} / \mathrm{Z}$ refers to the phase of $T_{A} / T_{B} / T_{C}$ for a given choice of $n_{p}$ and $\theta_{L}$. Obviously, when $n_{p}$ is very low, all of $T_{A}, T_{B}, T_{C}$ will be in their LD phases for any values of $\theta_{L}$ and thus the system adopts the phase LD-LD-LD. Similarly, for a very high $n_{p}$ all the channels will be in their HD phases and the system is in its HD-HD-HD phase. We now discuss the admissibility of the intermediate phases as $n_{p}$ varies. Bulk current conservation in the steady states, for any value of $\theta_{L}$, yields (in the MFT),

$$
\rho_{A}(x)\left[1-\rho_{A}(x)\right]+\rho_{B}(x)\left[1-\rho_{B}(x)\right]=\rho_{C}(x)\left[1-\rho_{C}(x)\right] .
$$

The maximum of the right hand side of Eq. (11) is 1/4, corresponding to the MC phase in $T_{C}$. This immediately rules out MC phases in $T_{A}$ or $T_{B}$ for $\theta_{L} \neq 0,1$. Thus, phases $(X, M C, Z)$ or $(M C, Y, Z)$ are not allowed. In addition, phases LD-LD-HD and HD-HDLD are prohibited for any $\theta_{L} \neq 0$ or 1 . If $T_{C}$ is in its MC phase for a given $\left(n_{p}, \theta_{L}\right)$ value, increasing $n_{p}$ will lead to addition of more particles with no change in $\rho_{C}(x)=1 / 2$ in the bulk. Hence, the extra particles should accumulate in either $T_{A}$ or $T_{B}$ or both, without any change in the total current given by Eq. (1). Hence, any addition of particles is then expected to manifest in the form of LDWs in $T_{A}$ or $T_{B}$ or both, which leave the currents unchanged. Our detailed steady state density profiles confirm this physically intuitive expectation.

The results from Model I are summarized in the phase diagram Fig. 2 where different phases are marked in the $\left(n_{p}, \theta_{L}\right)$-plane. The phase diagram is quite complex in having

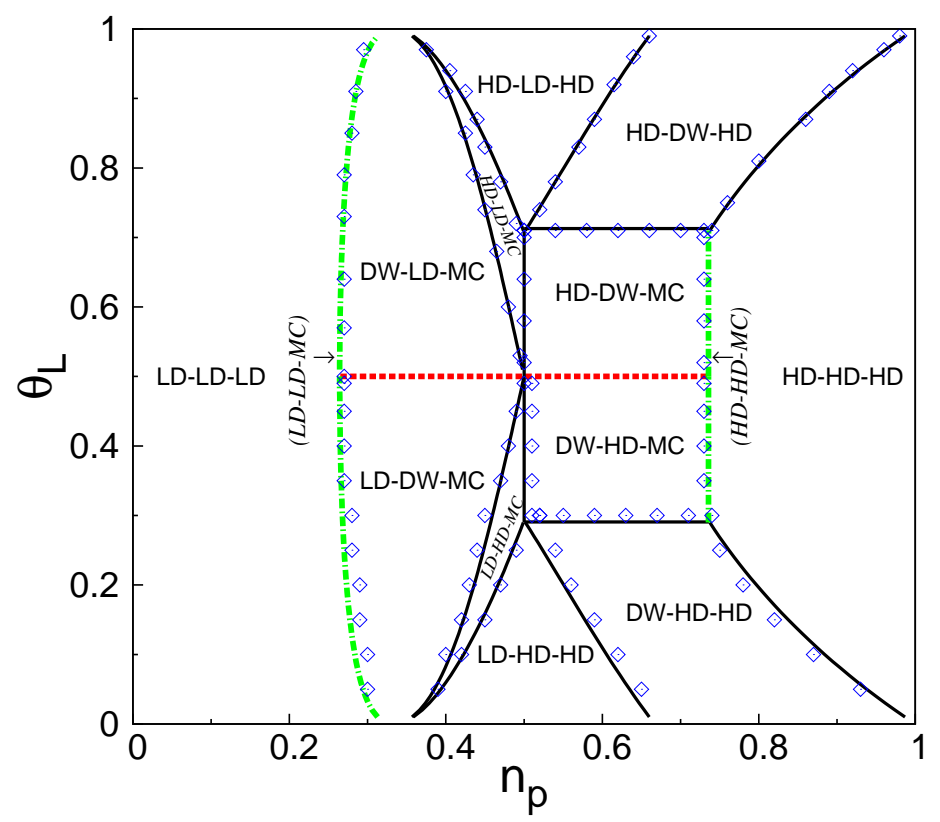

Figure 2. (Color online) Phase diagram in $\left(n_{p}, \theta_{L}\right)$-plane for Model I, phase boundaries are represented by black continuous lines, the line-phases are denoted by green dashed-dotted lines and DDW appears on red dotted line as obtained from MFT, whereas corresponding data points are from MCS with $N=500$.

a large number of phases, as expected. Notice that it is symmetric about the line $\theta_{L}=1 / 2$, a consequence of the interchange between $T_{A}$ and $T_{B}$ with an interchange 
between $\theta_{L}$ and $\left(1-\theta_{L}\right)$. Notice that in (2) some of the phases are represented by finite areas, whereas others appear just as lines. We discuss the physical principles for the calculation of the density profiles below with some illustrative examples. Full calculations of the steady state densities $\rho_{A}, \rho_{B}, \rho_{C}$ for Model II are given in Appendix as reference.

Consider the phase HD-DW-HD, with an LDW in $T_{B}$ at $x_{B}^{w}$ as shown in Fig. 3. Since $T_{A}$ and $T_{C}$ are in their HD phases, their densities are $\rho_{A}=1-\beta_{A}$ and $\rho_{C}=1-\beta_{C}$, neglecting the boundary layers $(\mathrm{BL})$. At $R J$, both $T_{A}$ and $T_{B}$ have no BL; current conservation at $R J$ yields $\beta_{A}=\beta_{B}$. Noting that there is an LDW in $T_{B}$, current conservation at $L J$ gives,

$$
\beta_{B}\left(1-\beta_{B}\right)=\left(1-\beta_{C}\right)\left(1-\theta_{L}\right) \beta_{B} .
$$

Overall current conservation in the bulk gives,

$$
\beta_{A}\left(1-\beta_{A}\right)+\beta_{B}\left(1-\beta_{B}\right)=\beta_{C}\left(1-\beta_{C}\right) .
$$

Using particle number conservation and neglecting BLs in TL we have,

$$
3 n_{p}=1-\beta_{A}+\beta_{B}+\left(1-x_{B}^{w}\right)\left(1-2 \beta_{B}\right)+1-\beta_{C} .
$$

The boundary lines between HD-LD-HD and HD-DW-HD phases and HD-DW-HD and HD-HD-HD phases may be obtained respectively by setting $x_{B}^{w}=1$ and $x_{B}^{w}=0$;

$$
\begin{aligned}
& 3 n_{p}=3-\beta_{A}-\beta_{B}-\beta_{C}, \\
& 3 n_{p}=2-\beta_{A}+\beta_{B}-\beta_{C} .
\end{aligned}
$$

However, these boundary lines do not span over the entire range $0 \leq \theta_{L} \leq 1$, but get cut off at $\theta_{L}<1$ at which another phase HD-DW-MC appears; see Fig. 3 for the corresponding density profiles. The phase boundary between HD-DW-HD and HD-DW$\mathrm{MC}$ is obtained by setting $\beta_{C}=1 / 2$. Now putting that in Eqs. (2) and (3) we have the equation of the horizontal boundary line as $\theta_{L}=1 / \sqrt{2}$.

The other boundaries of HD-DW-MC phase may be obtained as follows. Current conservation at the $R J$ gives $\beta_{A}=\beta_{B}$. Again from overall current in this phase yields

$$
\beta_{A}\left(1-\beta_{A}\right)+\beta_{B}\left(1-\beta_{B}\right)=1 / 4 \text {. }
$$

Considering $T_{A}$ in its HD phase, we get $\beta_{A}=0.146=\beta_{B}$ from Eq. (6) . Following the calculation logic outside above, the boundary lines between HD-LD-MC and HD-DWMC phases and HD-DW-MC and HD-HD-MC phases obtain respectively as

$$
\begin{aligned}
& 3 n_{p}=3 / 2, \\
& 3 n_{p}=5 / 2-2 \beta_{A} .
\end{aligned}
$$

The phase HD-DW-MC Again, HD-DW-MC does not span over the whole range of $\theta_{L}$ but gets cut by another phase DW-HD-MC as shown in the phase diagram.

In the special case with $\theta_{L}=1 / 2, \rho_{A}$ and $\rho_{B}$ should be statistically symmetric. Therefore, if one of them satisfies the condition for an LDW, the other also must satisfy the same. Thus, we should find a pair of domain walls one each in $T_{A}$ and $T_{B}$, at 

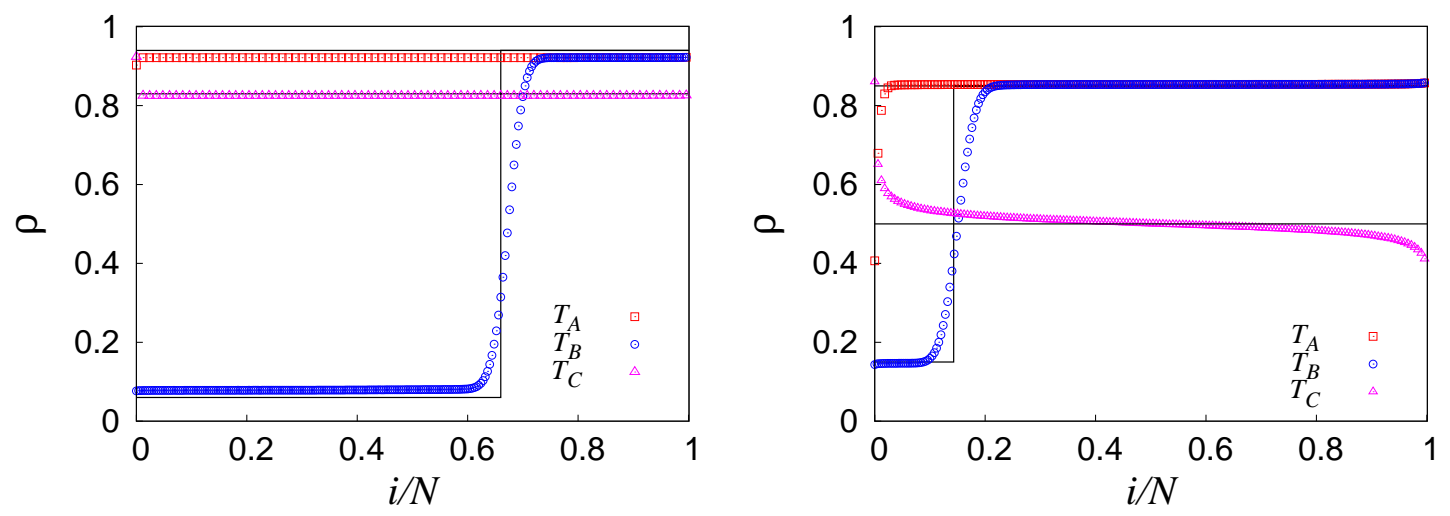

Figure 3. (Color online) (left) Density profiles for $T_{A}, T_{B}, T_{C}$ obtained with $n_{p}=0.70$, $\theta_{L}=0.90$ and $N=500$, displaying (HD-DW-HD) phase. LDW is found at $x_{B}^{w}=0.661$ from MFT which matches with the numerical value 0.672. (right) Density profiles for (HD-DW-MC) phase obtained with $n_{p}=0.70, \theta_{L}=0.55$ and $N=500$. LDW is found at $x_{B}^{w}=0.143$ from MFT which matches with the numerical value 0.149 . Numerical data displayed by points whereas solid lines denote MFT results.

locations $x_{w}^{A}$ and $x_{w}^{B}$, respectively. If there are indeed two LDWs in $T_{A}$ and $T_{B}$, then $\alpha_{A}=\beta_{A}=\alpha_{B}=\beta_{B}$. Furthermore, in this case $T_{C}$ must be in its MC phase, since once the LDWs are formed in $T_{A} / T_{B}$, any addition of particles in the system will lead to shifting of the LDW positions keeping the currents same. Using current conservation in the bulk, we have,

$$
\alpha_{A}\left(1-\alpha_{A}\right)+\alpha_{B}\left(1-\alpha_{B}\right)=1 / 4,
$$

solving which we have $\alpha_{A}=0.146=\alpha_{B}$.

Now from particle number conservation we get,

$$
3 n_{p}=\alpha_{A}+\left(1-x_{A}^{w}\right)\left(1-2 \alpha_{A}\right)+\alpha_{B}+\left(1-x_{B}^{w}\right)\left(1-2 \alpha_{B}\right)+1 / 2 .
$$

Thus, the LDW positions $x_{A}^{w}$ and $x_{B}^{w}$ can no longer be determined uniquely. Since, all (pairwise) values of $x_{A}^{w}$ and $x_{B}^{w}$ that satisfy Eq. (9), LDWs at each of such pairs of solutions for $x_{A}^{w}$ and $x_{B}^{w}$ are physically valid solutions for the density profiles in $T_{A}$ and $T_{B}$. In course of time the system should display all these solutions, over long time averages $\rho_{A}$ and $\rho_{B}$ will essentially appear as two inclined lines, which are the envelopes of the allowed LDW solutions. In other words, we will observe two DDWs. Our MFT analysis and physical arguments for DDWs are verified by our MCS studies. Figure 2 shows the DDW-DDW-MC line (red dotted line) as a borderline between the DW-LD$\mathrm{MC}$ and LD-DW-MC phases. In addition, representative DDW profiles for $\rho_{A}$ and $\rho_{B}$ are shown in Fig. 4 ,

Two DDWs instead of LDWs for $\theta_{L}=1 / 2$, can be argued from particle number conservation. Notice that for an LDW, its position is uniquely determined by the particle number conservation; see, e.g., Eq. (4) that gives $x_{B}^{w}$, the position of the LDW in $T_{B}$. However, if there are two LDWs in the system, it is clear that an arbitrary shift in the position of one of the LDWs, together with a compensating reverse shift of the position 


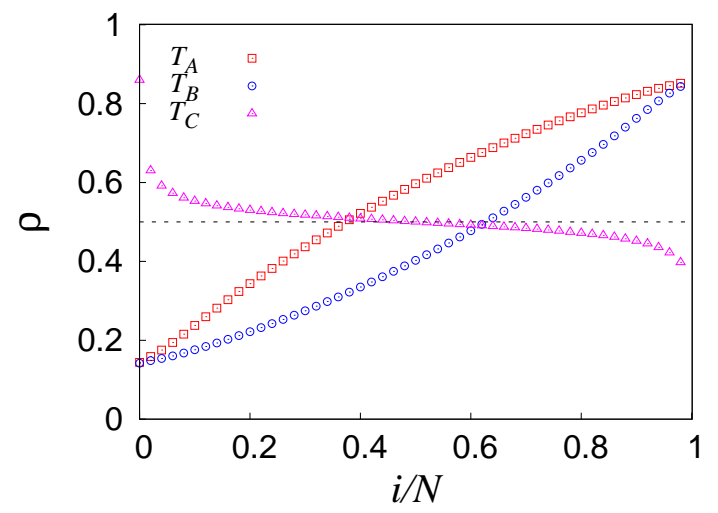

Figure 4. (Color online) Density profiles for $T_{A}, T_{B}, T_{C}$ obtained numerically with $\theta_{L}=0.50, n_{p}=0.40$ and $N=500$, displaying DDW's in $T_{A}$ and $T_{B}$ with MC phase in $T_{C}$.

of the second LDW keeps the total particle number conserved. Thus, the LDW positions are not uniquely determined. Hence the model displays DDWs. This also explains why for a single LDW, its position in the steady state is a quantity that remains stable by the dynamics of the system, where as for DDWs, it is the sum of their positions that remain stable.

Understandably, for a very low (high) $\theta_{L}, T_{A}\left(T_{B}\right)$ is in its LD phase and $\rho_{B}>(<) \rho_{A}$ in general. For a fixed $\theta_{L}$. when $n_{p}$ is very low, all of $T_{A}, T_{B}, T_{C}$ are in the LD phases, regardless of the value of $\theta_{L}$. As $n_{p}$ increases, $T_{B}$ and $T_{C}$ move to their DW/HD phase and $T_{C}$ to MC phases, while $T_{A}$ remains in its LD phase for small $\theta_{L}$. This is due to the fact that for a small $\theta_{L}$, very few particles enter $T_{A}$ in comparison with $T_{B}$, regardless of $n_{p}$. Eventually, as $n_{p}$ approaches unity (the system is nearly filled), all of $T_{A}, T_{B}, T_{C}$ should be in their HD phases, for any $\theta_{L}$. In general, the densities for $T_{A}, T_{B}, T_{C}$ always change continuously across the phase boundaries. Hence, with channel densities as the order parameter, the transitions are second order in nature.

The phases LD-LD-MC and HD-HD-MC are just lines as represented by green dashed-dotted lines in Fig. 2. The fact that they do not cover any area in the $\left(n_{p}, \theta_{L}\right)$ -plane can be understood in simple physical terms. Since for these phases, $T_{C}$ is in MC phase, $\rho_{C}=1 / 2$ and the current through it is $1 / 4$. Thus any putative change in $n_{p}$ is to be reflected by appropriate changes in $\rho_{A}$ and $\rho_{B}$. Since $T_{A}$ and $T_{B}$ are assumed to have uniform densities (LD or HD phases), any change in $n_{p}$ automatically leads to changes in $\rho_{A}$ and $\rho_{B}$ with associated changes in their currents as well. This in turn spoils the bulk current conservation, as the sum of their currents must be $1 / 4$ (= current in $T_{C}$ ). Thus, these particular phases can be realized only for one value of $n_{p}$ for a given $\theta_{L}$, which explains why they appear as lines. For instance, when both $T_{A}$ and $T_{B}$ are in their LD phases with densities $\alpha_{A}$ and $\alpha_{B}$, respectively, with $\alpha_{A} /\left(1-\alpha_{A}\right)=\theta_{L} /\left(1-\theta_{L}\right)$, the bulk currents here satisfy the same Eq. 8. Now, if $n_{p}$ is changed, say increased, then both $\alpha_{A}$ and $\alpha_{B}$ rise keeping their ratio unchanged. This, however, will spoil Eq. 8 . Hence, these phases can survive only for one particular value of $n_{p}$ for a given $\theta_{L}$, which 
is consistent with their appearance as a line in phase diagram Fig. 2 ,

\section{Summary and outlook}

To summarise, Model I reveals interesting interplay between multiple links connecting non-identical junctions and the junctions themselves, that determines the resulting macroscopic steady states density profiles of the overall closed system. Since $T_{C}$ is effectively an extended bottleneck in the system, our results in fact show the role of extended bottleneck in controlling the phases. Our scheme of MFT for obtaining the steady state density profiles by using current and total particle number conservations are generic and may be extended in straightforward ways to more complex closed systems having larger number of junctions and branches. As discussed in Sec. Appendix Abelow, an additional point defect in Model II brings in new macroscopic steady state behaviour, including the possibility of an LDW in $T_{C}$ and non-overlapping DDWs in $T_{A}$ and $T_{B}$. The steady state bulk current in each of the segments may be easily determined from the knowledge of the corresponding densities; thus we show how the steady state currents may be controlled by the extended and point bottlenecks. Useful modifications in the context of vehicular transport includes unequal lengths of the channels, allowing unequal channel lengths and different velocities for different particles and/or along different channels, impurities on the tracks and possibilities of change in speeds (acceleration and braking) [15]. Other possible relevant extensions of theoretical interests include (i) one or some of the branches allow bidirectional motion [10, 16], (ii) when there are local particle non conserving processes, e.g., random attachment and detachment of particles [17], and (iii) the presence of active and inactive agents (particles) [18]. We hope our work will motivate further works along these lines.

\section{Appendix A. Model II}

We now introduce Model II, a generalization of Model I, that now includes a point defect at the $R J$, defined by two new parameters $\theta_{R A}$ and $\theta_{R B}$; see Fig. A1. Here,

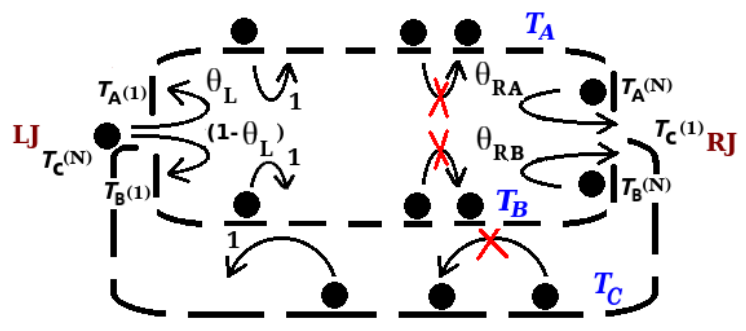

Figure A1. (Color online) Schematic diagram of Model II with two additional parameters $\theta_{R A}$ and $\theta_{R B}$ at the $R J$. 
$\theta_{R A}$ and $\theta_{R B}$ control the effective particle flow into the the two channels $T_{A}$ and $T_{B}$ as point defects at the junction position of regular TASEP channels. Obviously, if we set

$\theta_{R A}=1=\theta_{R B}$, Model II should reduce structurally to Model I. Analysis of the phases in Model II and their differences with their counterparts in Model I allows us to draw conclusions about the effects of the point defects at $R J$. As a model for vehicular traffic in a network of roads, these may potentially model dynamic obstacles (e.g., a traffic signal or sudden pedestrian crossings) at $R J$ in a simple way. We make a detailed comparison between Model I and Model II in Appendix Appendix D. To reduce the number of tunable parameters, we set $\theta_{R A}=1-\theta_{R B}=\theta_{R}$ (say), and thereafter all results for Model II are obtained keeping this relation.

\section{Appendix B. Phase diagram for Model II}

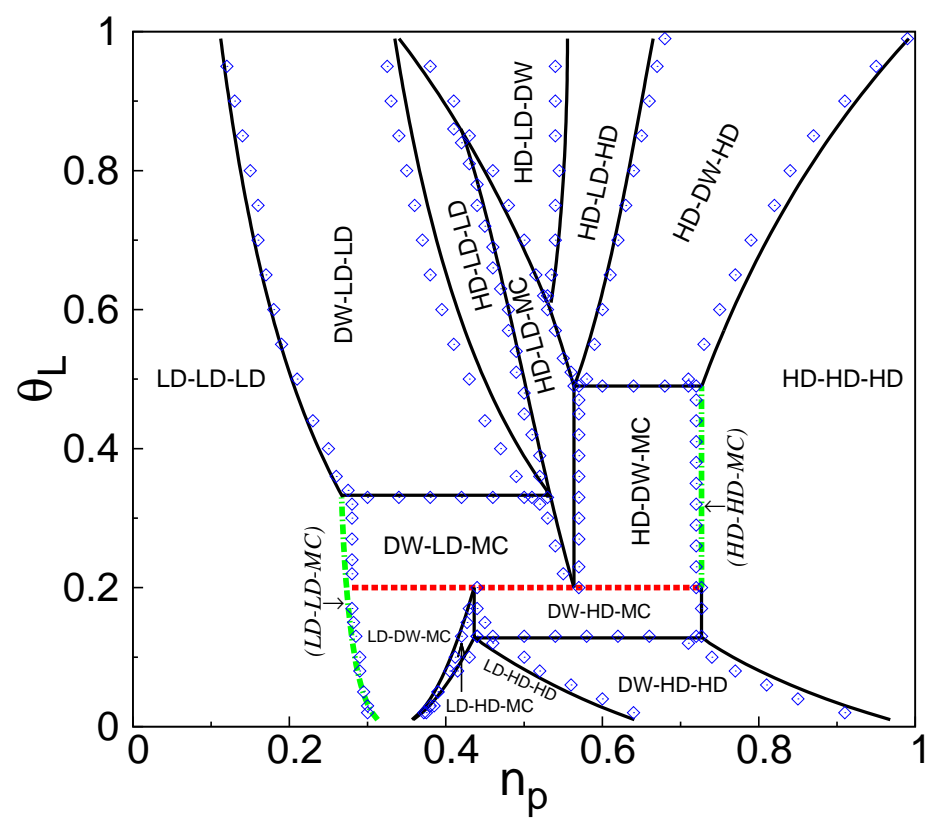

Figure B1. (Color online) Phase diagram in $\left(n_{p}, \theta_{L}\right)$-plane for Model II with $\theta_{R}=0.20$, phase boundaries are represented by black continuous lines, the line-phases are denoted by green dashed-dotted lines and DDW appears on red dotted line as obtained from MFT, whereas corresponding data points are from MCS with $N=500$.

The phase diagram for Model II is shown for $\theta_{R}=0.20$. This is quite different from the phase diagram of Model I in Fig. 2 regarding the structure and the total number of phases, as new phases emerge in comparison with Model I. 


\section{Appendix C. Density profiles for Model II}

Appendix C.1. $T_{A}$ in LD-HD coexisting phase, $T_{B}$ and $T_{C}$ both in $L D$ phase

Here, $\rho_{B}=\alpha_{B}, \rho_{C}=\alpha_{C}$ in the bulk, $\rho_{A}=\alpha_{A}$ near $L J$ and $\rho_{A}=1-\beta_{A}$ near $R J$ with $\alpha_{A}=\beta_{A}$, which meet in the bulk to form an LDW; see Fig. C1. At $L J$ and $R J$ the current conservation yields,

$$
\begin{aligned}
& \frac{\alpha_{A}}{\alpha_{B}}=\frac{\theta_{L}}{1-\theta_{L}}, \\
& \beta_{A}\left(1-\beta_{A}\right)=\left(1-\beta_{A}\right)\left(1-\alpha_{C}\right) \theta_{R} .
\end{aligned}
$$

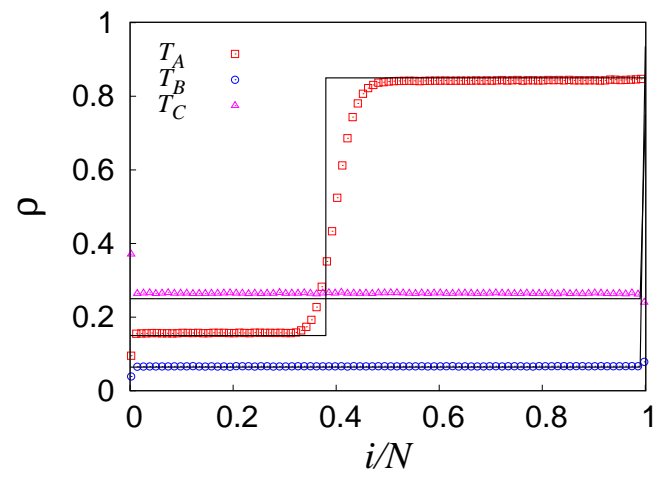

Figure C1. (Color online) Density profile for the phase DW-LD-LD with $n_{p}=$ $0.30, \theta_{L}=0.70, \theta_{R}=0.20$.

Now particle number conservation yields (neglecting BLs),

$$
3 n_{p}=\alpha_{A}+\left(1-x_{A}^{w}\right)\left(1-\alpha_{A}-\beta_{A}\right)+\alpha_{B}+\alpha_{C},
$$

where $x_{A}^{w}$ is the position of the LDW $\left(0<x_{A}^{w}<1\right)$ in $T_{A}$. From Eqs. A-1 $A-2$ $\alpha_{A}=\beta_{A}, \alpha_{B}, \alpha_{B}$ may be solved. The boundaries between the LD, LD-HD phases and LD-HD, HD phases of $T_{A}$ (with $T_{B}$ and $T_{C}$ in their LD phases) are obtained by setting $x_{A}^{w}=0$ and $x_{A}^{w}=1$, respectively in Eq.(A-2), as shown in Fig. B1 for $\theta_{R}=0.20$.

\section{Appendix C.2. $T_{A}$ in $L D-H D$ coexisting phase, $T_{B}$ in $L D$ and $T_{C}$ in $M C$ phase}

In this case, in the bulk $\rho_{C}=1 / 2, \rho_{B}=\alpha_{B} ; T_{A}$ continues to have an LDW at $x_{A}^{w}$ as before. Use now current conservation to write,

$$
\begin{aligned}
& \frac{\alpha_{A}}{1-\alpha_{A}}=\frac{\theta_{L}}{1-\theta_{L}}, \\
& \alpha_{A}\left(1-\alpha_{A}\right)+\alpha_{B}\left(1-\alpha_{B}\right)=1 / 4 .
\end{aligned}
$$

Now from particle number conservation we have,

$$
3 n_{p}=\alpha_{A}+\left(1-x_{A}^{w}\right)\left(1-\alpha_{A}-\beta_{A}\right)+\alpha_{B}+1 / 2 .
$$




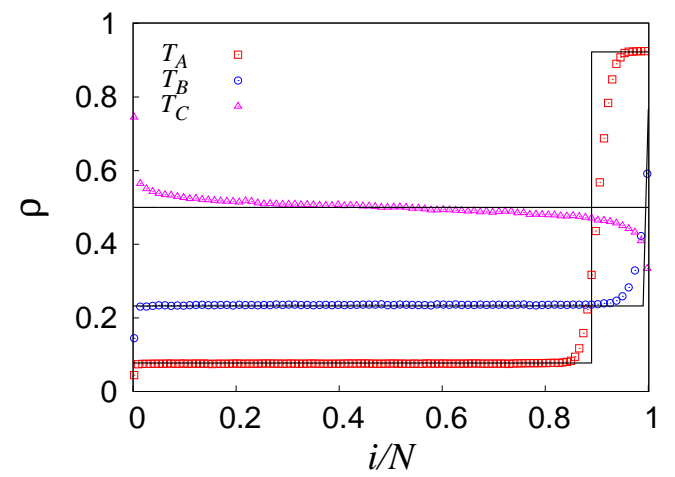

Figure C2. (Color online) Density profile for the phase DW-LD-MC with $n_{p}=$ $0.30, \theta_{L}=0.25$.

Equations (A-3, A-4 yield $\alpha_{A}, \alpha_{B}$ and $x_{A}^{w}$. The phase boundaries between LD, LD-HD and LD-HD, HD phases of $T_{A}$ are obtained by setting $x_{A}^{w}=0$ and $x_{A}^{w}=1$ respectively with $T_{B}$ in $\mathrm{LD}$ and $T_{C}$ in $\mathrm{MC}$ phase, and are shown in the phase diagram Fig. B1.

Appendix C.3. $T_{A}$ in $L D-H D$ coexisting phase, $T_{B}$ and $T_{C}$ both in $H D$ phase

In this case $\rho_{B}=1-\beta_{B}$ and $\rho_{C}=1-\beta_{C}$ in the bulk, and $T_{A}$ has an LDW. Thus $\alpha_{A}=\beta_{A}$. From current conservation

$$
\begin{aligned}
& \frac{\beta_{A}}{\beta_{B}}=\frac{\theta_{R}}{1-\theta_{R}}, \\
& \alpha_{A}\left(1-\alpha_{A}\right)=\left(1-\alpha_{A}\right) \theta_{L}\left(1-\beta_{C}\right) .
\end{aligned}
$$

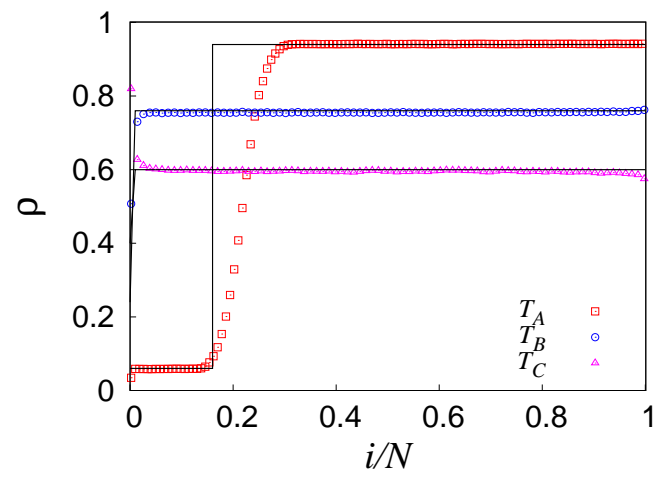

Figure C3. (Color online) Density profile for the phase DW-HD-HD with $n_{p}=$ $0.70, \theta_{L}=0.10, \theta_{R}=0.20$.

Particle number conservation yields

$$
3 n_{p}=\alpha_{A}+\left(1-x_{A}^{w}\right)\left(1-\alpha_{A}-\beta_{A}\right)+1-\beta_{B}+1-\beta_{C} .
$$


where $0<x_{A}^{w}<1$ is the position of the LDW in $T_{A}$. Eqs. (A-5 A-6 yield $\beta_{C}, \alpha_{A}$ and $x_{A}^{w}$. The phase boundaries between LD,LD-HD and LD-HD,HD phases of $T_{A}$ are, as usual, obtained by setting $x_{A}^{w}=0$ and $x_{A}^{w}=1$ respectively with both $T_{B}$ and $T_{C}$ are in their HD phases. For $\theta_{R}=0.20$ the boundaries are shown in Fig. B1.

Appendix C.4. $T_{A}$ in LD-HD coexisting phase, $T_{B}$ in $H D$ and $T_{C}$ in $M C$ phase

Here $\rho_{C}=1 / 2$ in the bulk. Current conservation at $R J$ gives,

$$
\frac{\beta_{A}}{\beta_{B}}=\frac{\theta_{R}}{1-\theta_{R}} \text {. }
$$

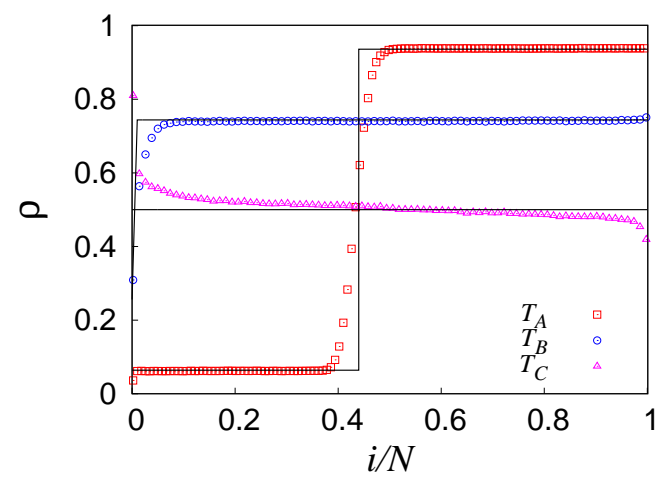

Figure C4. (Color online) Density profile for the phase DW-HD-MC with $n_{p}=$ $0.60, \theta_{L}=0.15, \theta_{R}=0.20$.

From particle number conservation we have,

$$
3 n_{p}=\alpha_{A}+\left(1-x_{A}^{w}\right)\left(1-\alpha_{A}-\beta_{A}\right)+1-\beta_{B}+1 / 2 .
$$

From Eq. A-7 and A-8 we $\alpha_{A}, \beta_{B}$ and $x_{A}^{w}$. The phase boundaries between LD,LD-HD and LD-HD,HD phases are obtained by setting $x_{A}^{w}=0$ and $x_{A}^{w}=1$ respectively with $T_{B}$ in $\mathrm{HD}$ and $T_{C}$ in MC phase, and for $\theta_{R}=0.20$ the boundaries are shown in Fig. B1.

Appendix C.5. $T_{A}$ in HD, $T_{B}$ in LD-HD coexisting phase and $T_{C}$ in $H D$ phase

In this case $\rho_{A}=1-\beta_{A}$ and $\rho_{C}=1-\beta_{C}$, respectively. Since $T_{B}$ is assumed to be in its LD-HD coexistence phase thus $\alpha_{B}=\beta_{B}$. From the current conservation we have,

$$
\begin{aligned}
\alpha_{B} & =\left(1-\beta_{C}\right)\left(1-\theta_{L}\right), \\
\frac{\beta_{A}}{\beta_{B}} & =\frac{\theta_{R}}{1-\theta_{R}} .
\end{aligned}
$$

Again from particle number conservation,

$$
3 n_{p}=1-\beta_{A}+\alpha_{B}+\left(1-x_{B}^{w}\right)\left(1-\alpha_{B}-\beta_{B}\right)+1-\beta_{C} .
$$

Here $0<x_{B}^{w}<1$ is the position of the localised DW in $T_{B}$, while $T_{A}$ and $T_{C}$ both are in HD phase. Eq. (A-9 A-10) yield $\alpha_{B}, \beta_{C}$ and $x_{B}^{w}$. The phase boundaries are obtained by setting $x_{B}^{w}=0$ and $x_{B}^{w}=1$, and for $\theta_{R}=0.20$ the boundaries are shown in Fig. B1. 


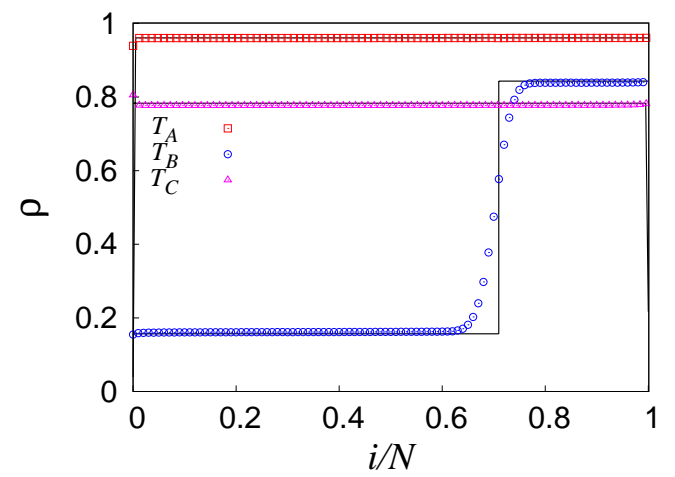

Figure C5. (Color online) Density profile for the phase HD-DW-HD with $n_{p}=$ $0.70, \theta_{L}=0.80, \theta_{R}=0.20$.

Appendix C.6. $T_{A}$ in $H D, T_{B}$ in $L D$ and $T_{C}$ in $L D-H D$ coexisting phase

In this case $\rho_{A}=1-\beta_{A}$ and $\rho_{B}=\alpha_{B}$ in the bulk, and $T_{C}$ is in DW phase. Thus $\alpha_{C}=\beta_{C}$, and $1-\beta_{C}$ give the bulk densities on the entry $(R J)$ and exit $(L J)$ sides of $T_{C}$. Now the current matching conditions at $L J$ and $R J$ give,

$$
\begin{aligned}
& \alpha_{B}=\left(1-\beta_{C}\right)\left(1-\theta_{L}\right), \\
& \beta_{A}=\theta_{R}\left(1-\alpha_{C}\right) .
\end{aligned}
$$

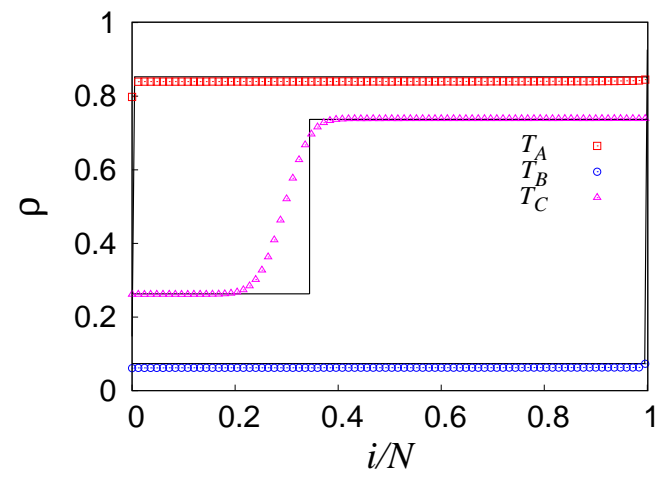

Figure C6. (Color online) Density profile for the phase HD-LD-DW with $n_{p}=$ $0.50, \theta_{L}=0.90, \theta_{R}=0.20$.

Now from particle number conservation we have,

$$
3 n_{p}=1-\beta_{A}+\alpha_{B}+\alpha_{C}+\left(1-x_{C}^{w}\right)\left(1-\alpha_{C}-\beta_{C}\right) .
$$

where $0<x_{C}^{w}<1$ is the position of the DW in $T_{C}$. From Eq. A-11 and $\mathrm{A}-12$ we get $\alpha_{C}, \alpha_{B}$ and $x_{C}^{w}$. The phase boundaries between LD,LD-HD and LD-HD,HD phases of $T_{C}$ are obtained by setting $x_{C}^{w}=0$ and $x_{C}^{w}=1$ respectively, and for $\theta_{R}=0.20$ the boundaries are shown in Fig. B1. 
Appendix C.7. Delocalised domain walls

Now, assume both $T_{A}$ and $T_{B}$ have domain walls. Then,we must have $\alpha_{A}=\beta_{A}$ and $\alpha_{B}=\beta_{B}$. Again from current matching conditions at $L J$ and $R J$ we have, $\alpha_{A} / \alpha_{B}=\theta_{L} /\left(1-\theta_{L}\right)$ and $\beta_{A} / \beta_{B}=\theta_{R} /\left(1-\theta_{R}\right)$ respectively. These gives the condition for having domain walls in both channels as,

$$
\theta_{L}=\theta_{R}
$$

By the similar argument as given for Model I we can show that, both the domain walls are delocalised with the sum of their positions remain stable and in that case $T_{C}$ remains in the MC phase. The density profiles for the channels are shown in Fig. C7, while the DDW boundary line is shown by red dotted line in phase diagram Fig. B1, Note that the DDWs in $T_{A}$ and $T_{B}$ are non-overlapping.

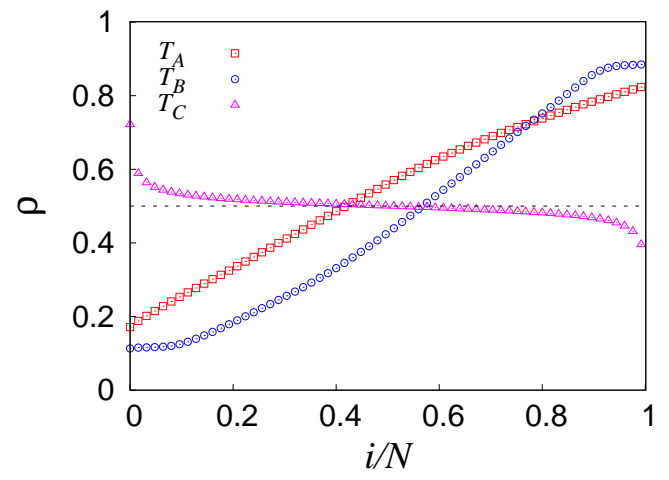

Figure C7. (Color online) Density profiles when both $T_{A}$ and $T_{B}$ has DDWs with $n_{p}=0.50, \theta_{L}=0.20=\theta_{R}$ and $N=500$.

\section{Appendix C.8. Boundary between DW-LD-LD and DW-LD-MC phases}

Here $\alpha_{C}=1 / 2$ at this phase boundary. Now from Eq. A-1 we have $\alpha_{A}=\theta_{R} / 2$ and using the overall current conservation the solutions for $\theta_{L}$ are given by,

$$
\theta_{L}=\frac{\theta_{R}^{2}}{\theta_{R}^{2}+\theta_{R} \pm \sqrt{\theta_{R}^{3}\left(2-\theta_{R}\right)}} .
$$

Clearly, $\theta_{L}$ is independent of $n_{p}$; for $\theta_{R}=0.20$ the boundary line is shown in the phase diagram Fig. B1.

\section{Appendix C.9. Boundary between DW-HD-MC and DW-HD-HD phases}

At the boundary $1-\beta_{C}=1 / 2$. Again from Eq. (A-7) we have $\alpha_{A}=\theta_{L} / 2$ and $\beta_{B}=q \alpha_{A}$, where $q=\left(1-\theta_{R}\right) / \theta_{R}$. Now putting these values in overall current conservation equation we have a quadratic equation in $\theta_{L}$ with the solutions given as,

$$
\theta_{L}=\frac{1+q \pm \sqrt{2 q}}{1+q^{2}}
$$


Now enforcing that $T_{B}$ is in its HD phase, Eq.(A-15) yields the phase boundary as a horizontal line in $\left(n_{p}, \theta_{L}\right)$-plane. For $\theta_{R}=0.20$ it is shown in Fig. B1.

Appendix C.10. Boundary between HD-DW-MC and HD-DW-HD phases

At the boundary $1-\beta_{C}=1 / 2$, and from Eq.(A-9) $\alpha_{B}=\left(1-\theta_{L}\right) / 2$ and $\beta_{A}=q^{\prime} \alpha_{B}$, where $q^{\prime}=\theta_{R} /\left(1-\theta_{R}\right)$. Now putting those values in the current conservation equation we have,

$$
\theta_{L}=\frac{1+q^{\prime} \pm \sqrt{2 q^{\prime}}}{1+q^{\prime 2}}
$$

Now enforcing HD phase for $T_{A}$, we have the phase boundary (a horizontal line) in the $\left(n_{p}, \theta_{L}\right)$-plane, as shown for $\theta_{R}=0.20$ in Fig. B1.

Appendix C.11. Boundary between HD-LD-MC and HD-LD-HD phases

At the transition $\beta_{C}=1 / 2$, and at $L J$ we have $\alpha_{B}=\left(1-\theta_{L}\right) / 2$. Now from overall current conservation,

$$
4 \beta_{A}\left(1-\beta_{A}\right)+\left(1-\theta_{L}\right)\left(1+\theta_{L}\right)=1 .
$$

From Eq. (A-17) and particle number conservation

$$
6 n_{p}=3-\theta_{L}+\sqrt{1-\theta_{L}^{2}}
$$

Equation (A-18) gives the corresponding phase boundary in the $\left(n_{p}, \theta_{L}\right)$-plane as shown Fig. B1.

Appendix C.12. Boundary between LD-HD-MC and LD-HD-HD phases

Set $\beta_{C}=1 / 2$, for this transition. Then at the $L J \alpha_{A}=\theta_{L} / 2$. From overall current conservation we write,

$$
4 \beta_{B}\left(1-\beta_{B}\right)+\theta_{L}\left(2-\theta_{L}\right)=1 .
$$

Particle number conservation together with Eq. (A-19) yield

$$
6 n_{p}=2+\theta_{L}+\sqrt{\theta_{L}^{2}-2 \theta_{L}} .
$$

Equation (A-20) gives the corresponding phase boundary in $\left(n_{p}, \theta_{L}\right)$-plane as shown in Fig. B1.

\section{Appendix D. Comparison between Model I and Model II}

We now compare and contrast the results from Model I and Model II. This will allow us to understand the extent to which the point defects at $R J$ can affect the phases. First of all, the phase diagram Fig. 2 for Model I is symmetric about the line $\theta_{L}=1 / 2$, whereas there is no such symmetry in the phase diagram Fig. B1 for Model II in general, due to the point defects at $R J$. 
In Model II there are some phases which has no analogue in Model I, for example DW-LD-LD phase. Let us consider this phase where the BLs are at the two opposite junctions, at $R J$ for $T_{B}$ and at $L J$ for $T_{C}$. At $R J$ the current matching conditions between $T_{A}$ and $T_{C}$ gives,

$$
\alpha_{A}\left(1-\alpha_{A}\right)=\left(1-\alpha_{A}\right)\left(1-\alpha_{C}\right) \theta_{R A} .
$$

Now use the condition for retrieving Model I from Model II given by $\theta_{R A}=\theta_{R B}=1$, then from Eq. (A-1) $\alpha_{A}=\left(1-\alpha_{C}\right)$. Put this in the overall current conservation equation, $\alpha_{A}\left(1-\alpha_{A}\right)+\alpha_{B}\left(1-\alpha_{B}\right)=\alpha_{C}\left(1-\alpha_{C}\right)$ to get, $\alpha_{B}\left(1-\alpha_{B}\right)=0$, implying $\alpha_{B}=0$ or 1 , which is unfeasible. Thus, the assumption of the existence of the phase DW-LD-LD in Model I is not correct; therefore, we conclude that DW-LD-LD phase does not appear for Model I. Similar arguments show that HD-LD-LD and HD-LD-DW phases are also absent for Model I. These observations are clearly validated by our MCS studies on Model I, as displayed in Fig. (2) . Evidently, the phases which appear only in Model II are solely due to the point defect at $R J$. Furthermore, the DDWs in Model I should be fully overlapping (under long time averages; not shown in Fig 4), a feature consistent with the symmetry of Model I about $\theta_{L}=1 / 2$. In contrast, the DDWs in Model II are generally non-overlapping (even under long time averages), due to the lack of any special symmetry in Model II as seen in Fig. C7. These differences are connected to the fact that in Model I by construction, DDWs in $T_{A}$ and $T_{B}$ correspond to $\alpha_{A}=\beta_{A}=\beta_{B}=\alpha_{B}$. In Model II however there are no such equalities, due to the presence of the point defect at $R J$. A related consequence is that Model I displays DDWs only for $\theta_{L}=1 / 2$, where as in Model II, it is possible to have DDWs for arbitrary $\theta_{L}$ so long as the general conditions for DDWs are met. Overall, thus, the effect of introducing point defects at $R J$ is not only to change the locations of the phases in the $\left(n_{p}, \theta_{L}\right)$ phase diagram qualitatively, but also to introduce new phases which were absent in Model I. Thus, the topology the phase diagram gets affected.

\section{Appendix E. Limiting cases of Model II}

The limiting cases of Model II reveal interesting features. For instance, when $\theta_{L}$ and $\theta_{R}$ are either 0 or 1 simultaneously then by construction either $T_{A}$ or $T_{B}$ is fully blocked. The remaining system then has equal hopping rate at every site. Thus, the average density at every site is just $n_{p}$. Furthermore, if say $\theta_{L}$ is 1 or 0 with $\theta_{R}$ having a value between zero and unity, then the junction $R J$ effectively serves as a point defect in an otherwise homogeneous ring executing TASEP. For instance, consider $\theta_{L} \rightarrow 1,0<\theta_{R}<1$, thus

eliminating $T_{B}$ and allowing for a point defect at $R J$, given by a reduced hopping rate $\theta_{R} \prime<1$. In this limit our model is identical to the model in Ref. [19] and our results in this limit $\theta_{L} \rightarrow 1$ are in agreement with that. 
Acknowledgement:- AB wishes to thank the Max-Planck-Gesellschaft (Germany) and Department of Science and Technology/Indo-German Science and Technology Centre (India) for partial financial support through the Partner Group programme (2009). A.K.C. acknowledges the financial support from DST (India) under the SERC Fast Track Scheme for Young Scientists [Sanction no. SR/FTP/PS-090/2010(G)].

\section{References}

[1] Chowdhury D, Santen L and Schadschneider A, 2000 Phys. Rep. 329, 199

[2] Helbing D, 2001 Rev. Mod. Phys. 73, 1067

[3] Zia R K P, Dong J J, and Schmittmann B, 2011 J. Stat. Phys. 144, 405; Neri I, Kern N and Parmeggiani A, 2011 Phys. Rev. Lett. 107, 068702

[4] Nagel K and Schreckenberg M, 1992 J. Phys. I 2, 2221

[5] Schutz G and Domany E, 1993 J. Stat. Phys. 72, 277

[6] Schutz G, 2000 Phase Transitions and Critical Phenomena, vol. 19, edited by C. Domb and J. Lebowitz (Academic Press, London).

[7] Ito H and Nishinari K, 2014 Phys. Rev. E 89, 042813

[8] Zhang J, Li X, Wang R, Sun X and Cui X, 2012 Physica A 391, 2381

[9] Zhang L, de Gier J and Garoni T M, 2014 Physica A 401, 82

[10] Chatterjee R, Chandra A K and Basu A, 2013 Phys. Rev. E 87, 032157

[11] Hinsch H and Frey E, 2006 Phys. Rev. Lett. 97, 095701; Jiang et al, 2011 Phys. Rev. Lett. 106, 079601; Hinsch et al 2011 Phys. Rev. Lett. 106, 079602

[12] Brankov J G, Pesheva N C and Bunzarova N, 2004 Phys. Rev. E 69, 066128; Pesheva N C and Brankov J G, 2012 Biomath 1, 1211211; Pesheva N C and Brankov J G, 2013 Phys. Rev. E 87, 062116

[13] Schmittmann B and Zia R K P, 1995 Phase Transitions and Critical Phenomena, vol. 17, edited by C. Domb and J. Lebowitz (Academic Press, London); Chou T, Mallick K and Zia R K P, 2011 Rep. Prog. Phys. 74, 116601

[14] Krug J, 1991 Phys. Rev. Lett. 67, 1882

[15] Schreckenberg M, Schadschneider A, Nagel K, and Ito N, 1995 Phys. Rev. E 51, 2939; Ziang R, Hu M, Nishinari K, Wang R and Wu Q, 2010 J. Stat. Mech. P07003; Lazo M J and Ferreira A A, 2012 J. Stat. Mech. P05017; Furtlehner C, Lasgouttes J -M, and Samsonov M, 2012 J. Stat. Phys. 147, 1113

[16] Muhuri S, Shagolsem L, and Rao M, 2011 Phys. Rev. E 84, 031921

[17] Parmeggiani A, Franosch T and Frey E, 2003 Phys. Rev. Lett. 90, 086601

[18] Pinkoviezky I and Gov N S, 2013 Phys. Rev. E 88, 022714

[19] Janowsky S A and Lebowitz J L, 1992 Phys. Rev. A 45, 618 